\title{
Al/CuFeS2/ITO BEHAVES LIKE SWITCH DEVICE WITH NEGATIVE DIFFERENTIAL RESISTANCE
}

\author{
AnimeshLayek ${ }^{1}$, SomnathMiddy ${ }^{2}$ \\ ${ }^{1}$ Department of Physics, Bejoy Narayan Mahavidyalaya, Itachuna, Hooghly-712147, India \\ ${ }^{2}$ Department of Physics, BankimSardar College, Tangrakhali, South 24-paraganas -743329, India
}

\begin{abstract}
Semiconducting $\mathrm{CuFeS} 2$ nanoparticle has been synthesized by hydrothermal technique. Its special physical and electronic properties through the nanostructures make it a promising material for versatile device application. Here a thin film device of configuration $\mathrm{Al} / \mathrm{CuFeS2/ITO}$ has been fabricated, which behaves like a resonant tunneling device. The device shows a negative differential resistance at room temperature. The characteristic of this portable device discloses its probable applicability in solidstate switch.
\end{abstract}

Keywords: $\mathrm{CuFeS}_{2}$; Nanomaterial; Synthesis; Characterization; Resonance tunneling; Negative resistance.

\section{INTRODUCTION}

Currently the nonlinear current-voltage characteristic of Metal-semiconductor junctions explores the field of electronics as well as creates a great interest to several researchers and scientists.For almost all semiconductors based electronic devices, the reliable and well-controlled contacts are needed. Metal-semiconductor junctions serve the purpose in the form of barrier diodes [1]. The structural property of such metal-semiconductor junction plays a vital role in the device performance [2] and is the most widely used in semiconductor electronic devices, such as field effect transistors, solar cells and photodetectors etc. [3, 4]. Moreover another interesting sandwich structure metal/semiconductor/metal junctions reveal overriding current-voltage characteristics with the new concept of negative differential resistance. Standard applications of the kind of effect include use in ultra-fast pulse forming, radiation detection and signal generation system. In semiconductor device one of the responsible mechanisms for the negative resistance effect is resonant tunneling. This differential resistive behavior in conventional semiconductor based resonant tunnel diode has been tuned by modifying the materials, dopant concentration and the physical configuration of the device. Several reports have examined the relationship between negative differential resistance and electro activity in organic and inorganic systems. However, control of parameters governing negative differential resistance behavior in inorganic semiconductor material is just beginning to be explored experimentally and theoretically.

It is extremely important to study the interface properties for better understanding of the electrical properties of the metalsemiconductor junction that mainly control the performance of the junction diode [4-6]. In general, the physical properties of the metal/semiconductor interface depend on the surface preparation methods, which ultimately affect the performance, electrical properties, stability and reliability of the metal-semiconductor based devices [7, 8]. A resonant tunnel diode consists of a quantum well separated from two electrical contacts by two tunnel barriers. In resonant tunneling, the tunnel barriers control the probability of electrons moving from one electrode to the other through the quantum well. In conventional semiconductor based resonant tunnel diode, NDR behavior has been tuned by modifying the materials. However, control of parameters governing NDR behavior in inorganic semiconductor material is just beginning to be explored experimentally. Here for the first time we obtained the resonant tunneling of charge carrier and NDR behavior for the sandwich structured ITO/CuFeS$/ 2$. In this article the electrical behavior of the sandwich structureITO/CuFeS $2 / \mathrm{Allhave}$ been illustrated aptly. In this art of work a new kind of $\mathrm{CuFeS}_{2}$ nanomaterial have been derived by hydrothermal technique. The underneath mechanism for the occurrence of such phenomena is illustrated on the focus of resonant tunneling, quantitatively.

\section{EXPERIMENTAL SECTION}

\subsection{Materials and Synthesis}

In typical synthesis of $\mathrm{CuFeS}_{2}$ nanomaterial, a mixture of 10 $\mathrm{ml}$ of $0.1 \mathrm{M} \mathrm{FeCl}_{3}$ and $10 \mathrm{ml}$ of $0.1 \mathrm{M} \mathrm{CuCl}_{2}$ was taken into a glass beaker. By continuing stirring technique solution of $0.1 \mathrm{~N} \mathrm{Na}_{2} \mathrm{~S}$ solution was added drop wise with this mixture till a dark brown precipitate (ppt) was obtained. Few drops of PVP [poly(vinyl-pyrrolidone)] were added as surfactant with the above mixture and stirred during precipitation (ppt). All the chemical reactions were carried out in open atmosphere. After collecting the ppt, it was transferred to a linear Teflon autoclave and was heated through overnight at temperature $140^{\circ} \mathrm{C}$. The baked ppt was collected and washed with ethanol and distilled water repeatedly and sequentially by centrifuge technique. The powder form of the synthesized material was collected and used for characterization by heating at $100^{\circ} \mathrm{C}$ inside a vacuum oven. 


\subsection{Fabrication of Thin Film as Sandwich}

\section{Structure}

The spin coating technique was used for depositing $\mathrm{CuFeS}_{2}$ thin film on pre-cleaned (by acetone, ethanol and distilled water sequentially) ITO coated glass substrate. In this regard dispersed solution of $\mathrm{CuFeS}_{2}$ was prepared in chloroform medium. The film was dried under vacuum desiccator for 48 hours. Finally the top aluminium electrode was deposited on the active thin layer by thermal evaporation technique with the help of vacuum coating unit (12A4D HHV, Vacuum coating unit).

\subsection{Physical Measurements}

The structural, electrochemical and electrical properties of $\mathrm{CuFeS}_{2}$ were characterized by Bruker D8 powder X-Ray Diffractometer, Field Emission Scanning Electron Microscopy (FESEM) technique,electrochemical measurements were carried out using a Gamry reference 750 potentiostat in a three-electrode configuration and Keithley 2400 sourcemeter respectively.

\section{RESULTS AND DISCUSSIONS}

Fig.1 illustrate the X-ray diffraction (XRD) pattern of the $\mathrm{CuFeS}_{2}$ powder. The responsible Bragg's diffraction peaks approved $\mathrm{CuFeS}_{2}$, which is supported by the JCPDS Card No: 01-0842 [9,10].In order to investigate the morphology of the synthesized $\mathrm{CuFeS}_{2}$, TEM image was recorded. Typical TEM image of the sample in the present study is shown in Fig. 2, depicts that the particles are nanoscale in size with coagulation.

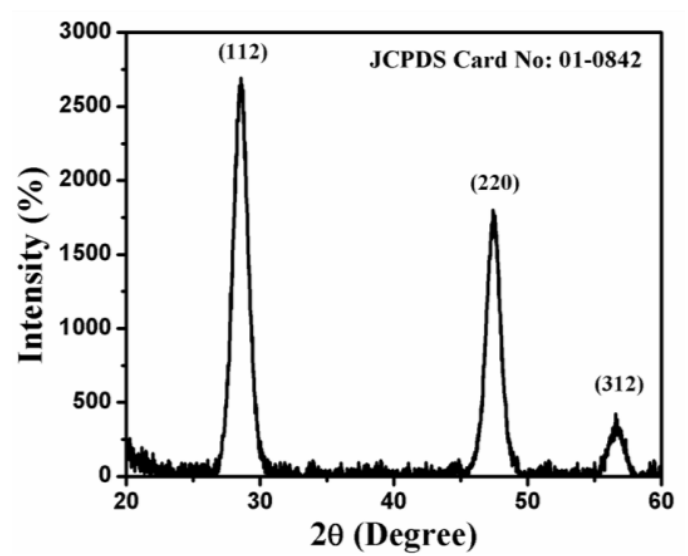

Fig. 1: XRD pattern of $\mathrm{CuFeS}_{2}$

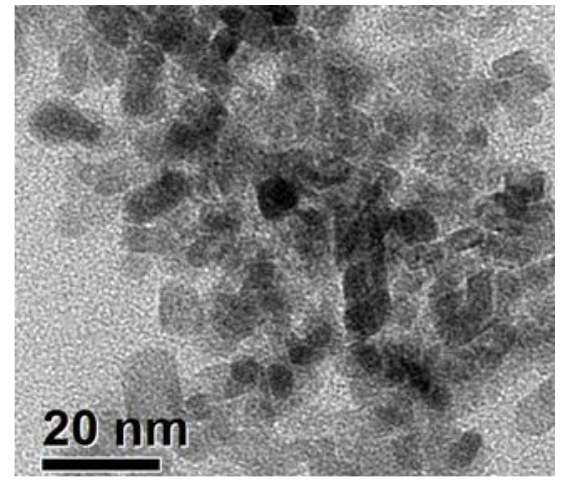

Fig. 2: TEM pattern of $\mathrm{CuFeS}_{2}$

The electrical conductivity of this nano-material was measured as $47.27 \times 10^{-9} \mathrm{Scm}^{-1}$ at room temperature by twoprobe technique.

The electro-chemical study was performed to determine the band position by investigating the onset state of oxidation and reduction of the material. The working electrode was a photocatalyst powder-modified glassy carbon electrode (GCE) (3 mm diameter), the reference electrode was an $\mathrm{Ag} / \mathrm{AgCl}$ (in $3 \mathrm{M} \mathrm{NaCl}$ ) electrode, and the counter electrode was a platinum wire in this respect. Fig. 3 shows the cyclic voltametry curve of dispersed $\mathrm{CuFeS}_{2}$ nanoparticles in chloroform medium.

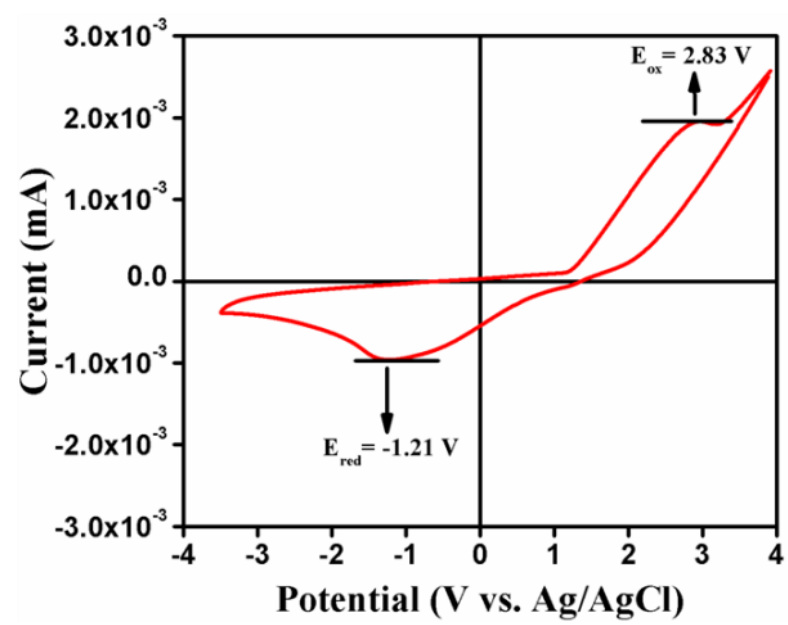

Fig. 3: HOMO-LUMO estimation from $\mathrm{C}-\mathrm{V}$

The voltamogram exhibits an oxidation peak (i.e., the ionization potential) at approximately $+2.83 \mathrm{~V}$ and a reduction peak (i.e., the electron affinity) at about $-1.21 \mathrm{~V}$. In order to findthe electrochemical band gap energy $(\Delta \mathrm{E})$, the conduction $\left(\mathrm{E}_{\mathrm{C} . \mathrm{B}}\right)$ and valence band $\left(\mathrm{E}_{\mathrm{V} . \mathrm{B}}\right)$ energy positions were estimated at $-2.93 \mathrm{eV}$ and $-6.97 \mathrm{eV}$ with the help of equations: $E_{V . B}=-\left(E_{o x}+4.14\right) e V$ and $E_{C . B}=-\left(E_{\text {red }}+\right.$ 4.14) eV.Where $E_{o x}$ and $E_{\text {red }}$ are the onset potentials of the oxidation and reduction relative to an $\mathrm{Ag}$ reference electrode. The value $4.14 \mathrm{eV}$ was considered for the difference between the vacuum level potential of the normal hydrogen electrode and the potential of the $\mathrm{Ag} / \mathrm{AgNO}_{3}$ electrode. Calculating the difference $\Delta \mathrm{E}=\mathrm{E}_{\mathrm{C} . \mathrm{B}}-\mathrm{E}_{\mathrm{V} . \mathrm{B}}$, the electrochemicalenergy band gap of $\mathrm{CuFeS}_{2}$ was evaluated as $4.04 \mathrm{eV}$. 
The above thermo-electrical characterization approved $\mathrm{CuFeS}_{2}$ as a promising semiconductor of wide band gap with conductivity of the ordered of $47.27 \times 10^{-9} \mathrm{Scm}^{-1}$ at room temperature. It is important to note that the band gap of our synthesized semiconductor material is $(=4.04 \mathrm{eV})$ and that of the work function of Aluminium $\left(\emptyset_{\mathrm{m}}=4.1 \mathrm{eV}\right)$, which basically can create an ideal background for a rectifying Schottky barrier when two systems are in contact with each other. In this approach we have made such contact to study the charge transport phenomena in between the metal-semiconductor junction by applying an external bias voltage. Our sincere approach involved us to record and analyzes the current-voltage (I-V) characteristic. Fig. 4 represented the I-V characteristic.

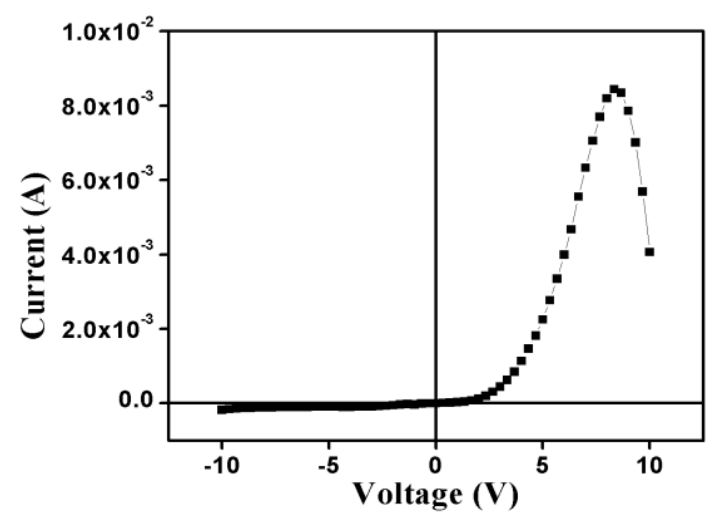

Fig. 4: Current vs Voltage (I-V) characteristic of the ITO/CuFeS ${ }_{2} / \mathrm{Al}$ junction

This current-voltage characteristic corresponds to resonance tunnel diode (RTD) [11]. The I-V characteristic curve (Fig. 5) represents three distinct regions with different slope. The slope at lower bias voltage indicates the tunneling effect of charge carriers that can be ascribed due to thermoionic emission. As the bias voltage increased the corresponding current increased spontaneously upto $8.3 \mathrm{~V}$ with increasing slope that can be ascribed by space-charge-limited-current (SCLC) theory. At high bias voltage the characteristic curve represented a negative differential resistance (NDR). The probable charge transport phenomenon for $\mathrm{ITO} / \mathrm{CuFeS}_{2} / \mathrm{Al}$ configuration can be illustrated as follow:

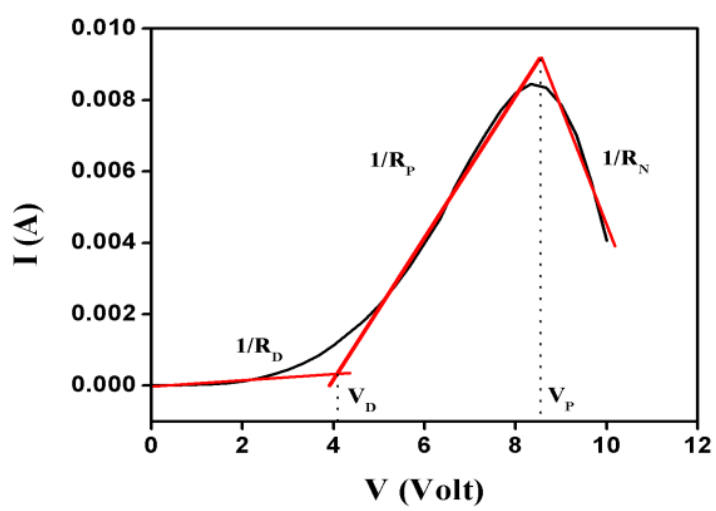

Fig. 5: Current-Voltage characteristic of the device with different slopes
$\mathrm{CuFeS}_{2} / \mathrm{Al}$ presented a structure where free electrons in $\mathrm{CuFeS}_{2}$ layer can exist at energies below the Fermi-level of Aluminium (work function $4.08 \mathrm{eV}$ ). Hence the Al layer acts as barrier to electron transport. On the other face $\mathrm{CuFeS}_{2} / \mathrm{ITO}$ presented a junction where free electrons in $\mathrm{CuFeS}_{2}$ layer can exist at energies (HOMO) below the Fermi-level of ITO (work function $4.4 \mathrm{eV}$ ). Consequently ITO layer forms another barrier to electron transport. For thin layer, electrons trapped within these barriers, approximately confined to discrete energy levels, as described by a simplistic quantum-mechanical model of a square well potential. Since the barriers are of finite width, these electrons would tend to leak out via quantum tunneling. Electrons from outside the well that hit the barrier structures will largely reflected, except those within a very narrow range around the discrete energy levels of the well, which acts as an electron energy filter. In real electronic applications, electrons have a thermally distributed range of energies. Applying an electric potential difference shifts the well's energy levels. As this energy level passes through the peak of the thermal electron distribution, the current peaks, giving a distinctive peak to the current voltage curve. In this region a positive static resistance $\left(R_{P}\right)$ plays its role to conduct current within the channel. In the region where the current decreased with increasing voltage, we have got a negative differential resistance $\left(\mathrm{R}_{\mathrm{N}}\right)$. For our device, in the forward bias direction of the I-V characteristic, the current increases first to a maximum value $I_{p}=8.4 \mathrm{~mA}$ (peak current) at a voltage $\mathrm{V}_{\mathrm{p}}=8.3 \mathrm{~V}$ (peak voltage), then decreases to a minimum value $4.0 \mathrm{~mA}$ at a voltage $\mathrm{V}_{\mathrm{v}}=10 \mathrm{~V}$. The diode negative differential resistance $\left(R_{N}\right)$ is estimated as $1.98 \mathrm{~K} \Omega$ from the equation $\mathrm{R}_{\mathrm{N}}=-2 \mathrm{~V}_{\mathrm{p}} / \mathrm{I}_{\mathrm{p}}$. In lower forward bias region the resistance that arises due to the current for thermoionic emission can be measured from the slope of lower voltage region and is illustrated as $\left(R_{D}\right)$ dynamic resistance of diode.

\section{CONCLUSION}

In conclusion, we demonstrate the experimental I-V characteristics of $\mathrm{CuFeS}_{2}$ for the configuration ITO/CuFeS $2 / \mathrm{Al}$, exhibits resonance-tunnelling behaviour with negative differential resistance at applied bias potential $\pm 10 \mathrm{~V}$. The carrier transport mechanisms are well established and approved by the determination of energy band position of $\mathrm{CuFeS}_{2}$ from the electrochemical methods. As a result from this interventional analysis of tunneling effect this structure can be applicable for solid-state switch.

\section{ACKNOWLEDGEMENT}

The authors acknowledge the support of ArkaDey of the Department of physics, Jadavpur University, Kolkata. This research was supported by a grant funded by the UGC minor research project of Delhi, India.

\section{REFERENCES}

[1]. S. M. Sze, Physics of Semiconductor Devices, $2^{\text {nd }}$ ed., (Wiley-Blackwell, New Jersey, USA,1981). 
[2]. M. Okutan, E. Basaran and F. Yakuphanoglu, Appl.Surf.Sci. 2521966 (2005).

[3]. R. L.V. Meirhaeghe, W. H. Laflere and F. Cardon, J. Appl. Phys. 76, 403 (1994).

[4]. E. Rhoderick and R. Williams, Metal-Semiconductor Contacts, (Oxford, Clarendon, 1988).

[5]. P. Hanselaer, W. Laflere, R. Van Meirhaeghe and F. Cardon, J. Appl. Phys. 56, 2309 (1984).

[6]. P. Hanselaer, W. Laflere, R. Meirhaeghe and F. Cardon, Appl. Phys. A: Mater. Sci. Process. 39, 129 (1986).

[7]. S. Karatas, C. Temirci, M. Cakar and A. Turut, Appl. Surf. Sci. 252, 2209 (2006).

[8]. M. E. Aydin, F. Yakuphanoglu, J. H. Eom and D. H. Hwang, Phys. B: Conden. Matter. 387, 239 (2007).

[9]. A. Layek, S. Middya, A. Dey, M. Das, J. Datta, C. Banerjee and P. P. Ray, J. Alloys and Compounds 613, 364 (2014).

[10]. K. A. Ngoen, T. Thongtem, S. Thongtem and A. Phuruangrat, Mater. Lett. 101, 9 (2013)

[11]. R. A. Wassel, G. M. Credo, R. R. Fuierer, D. L. Feldheim, and C. B. Gorman, J. Am. Chem. Soc. 126, 295 (2004) 